\title{
Development of a fast and reliable system for the automatic characterization of Giant magnetoimpedance samples
}

\author{
Eduardo C. Silva ${ }^{1}$, João H.C.C. Carneiro ${ }^{2}$, Luiz A.P. Gusmão ${ }^{1}$, Carlos R.H. Barbosa ${ }^{3}$, Elisabeth C. \\ Monteiro $^{3}$ \\ ${ }^{1}$ Department of Electrical Engineering, Pontifícia Universidade Católica do Rio de Janeiro, Rua Marquês de São Vicente 225, 22451-900 Rio \\ de Janeiro, Brazil \\ ${ }^{2}$ PEE - COPPE/UFRJ, Universidade Federal do Rio de Janeiro, Centro de Tecnologia - Bloco H, Sala 321, 21941-972 Rio de Janeiro, Brazil \\ ${ }^{3}$ Postgraduate Program in Metrology, Pontifícia Universidade Católica do Rio de Janeiro, Rua Marquês de São Vicente 225, 22451-900 Rio \\ de Janeiro, Brazil
}

\section{ABSTRACT}

Giant Magnetoimpedance (GMI) magnetometers are one of the most recent families of magnetic transducers, being characterized by their potential to achieve high sensitivities. The sensitivity of magnetic transducers is directly related to the sensitivity of its sensor elements. Thus, optimizing the sensitivity of these sensor elements is a critical part of the magnetometers development chain. This paper describes an automatic characterization system designed for the measurement of the electrical impedance of Giant MagnetoImpedance samples. The measurement uncertainties of the system were verified and discussed. The high speed of measurement attained with the use of this system, implemented in LabVIEW, allows for the rapid determination of the optimal operational point of GMI magnetometers.

Keywords: Giant Magnetoimpedance; Automatic Characterization System; Impedance Measurements; Magnetic Sensors

Citation: Eduardo C. Silva, João H.C.C. Carneiro, Luiz A.P. Gusmão, Carlos R.H. Barbosa, Elisabeth C. Monteiro, “Development of a fast and reliable system for the automatic characterization of Giant magnetoimpedance samples", Acta IMEKO, vol. 2, no. 1, article 8, August 2013, identifier: IMEKO-ACTA-02(2013)-0108

Editors: Paolo Carbone, University of Perugia, Italy; Ján Šaliga, Technical University of Košice, Slovakia; Dušan Agrež, University of Ljubljana, Slovenia Received January $10^{\text {th }}, 2013$; In final form March $16^{\text {th }}, 2013$; Published August 2013

Copyright: (C) 2013 IMEKO. This is an open-access article distributed under the terms of the Creative Commons Attribution 3.0 License, which permits unrestricted use, distribution, and reproduction in any medium, provided the original author and source are credited

Funding: This work was supported by the Brazilian funding agencies CNPq and FAPERJ

Corresponding author: Eduardo C. Silva, e-mail: edusilva@ele.puc-rio.br

\section{INTRODUCTION}

The successful use of Giant Magneto-Resistive (GMR) materials in the construction of precision magnetometers in the area of spintronics, in conjunction with the recent award of the 2007 Nobel Prize in Physics to GMR's co-inventors, Albert Fert and Peter Grünberg, has spurred much activity by research groups in the GMI effect.

Even though similarly named, the GMR and GMI effects differ in their nature. While GMR has explanations in quantum mechanics, GMI is derived from the skin effect. The GMI effect is a physical phenomenon characterized by large variations in the electrical impedance when soft amorphous ferromagnetic samples of specific composition are subjected to an external magnetic field [1-2].
In spite of the GMI effect being a recent discovery, GMI magnetometers have already been developed for various applications, among which stand out: presence detectors [3], industrial process control [4], space research and aerospace applications [1, 5], navigation systems [6], high-density memories and HDs [7], traffic control [8], cracks detection in materials [9], and biological and biomedical applications [10-16].

Researches in the GMI effect has shown that, with further sophistications, magnetometers based on GMI technology may have enough resolution to replace even SQUIDs (Superconducting Quantum Interference Devices), the currently most sensitive magnetometers [1, 5, 17-18], in some applications. In the area of biomagnetism, an eventual GMI magnetometer has promising potential uses, because of the resolution in the order of some picoteslas [15, 17-19]. 
The sensitivity of a magnetic transducer is directly related to the sensitivity of its sensor elements. Thus, the optimization of the sensitivity of the sensing elements is essential. However, to date there is no accurate mathematical model for the impact of the various physical parameters that influence the GMI effect. Thus, the optimization process is usually empirical [20]. Among that set of parameters, it can be mentioned: chemical composition and geometry of the sample (length, width and thickness); DC level, amplitude and frequency of the current used to excite the samples; components of the external magnetic field (longitudinal, orthogonal and perpendicular) and temperature [2].

In the research carried out by LaBioMet at PUC-Rio, aiming at defining the set of parameters that optimizes the sensitivity of the GMI sensor elements, the impact of various parameters is estimated via multiple experimental measurements. The large number of variables that should be analyzed in the measuring procedure motivated the development of an automatic characterization system, which accepts as input a list of measurement configurations, and outputs the resulting measurement data - readings of magnitude and phase of the impedance of GMI samples [21].

\section{GIANT MAGNETOIMPEDANCE}

In general, the impedance of a conductor depends on the distribution of the current inside the material. When the frequency increases, it is common for the current to concentrate in the surface of the conductor. In magnetic materials, the value of the current skin depth depends not only on the applied current amplitude and frequency value, but on the conductor geometry and on its magnetic permeability, which can vary with the applied magnetic field. This implies that, in samples of high permeability materials, even at moderate frequencies, a variation of the conductors' impedance with the value of the applied magnetic field can be expected [22].

In the GMI effect, the alternating current applied along the sample length creates a transverse magnetic field $\left(b_{a c}\right)$. This field magnetizes the material, increasing its permeability. The permeability grows until the external magnetic field $(H)$ becomes sufficiently high to rotate the magnetic domains of the sample along its direction. The permeability dependence with the magnetic field and with the current modifies the skin depth of the current inside the material and, consequently, the impedance of the sample [23-26]. Ribbons and wires of soft ferromagnetic alloys of the series $\mathrm{CO}_{75-\mathrm{x}} \mathrm{Fe}_{\mathrm{x}} \mathrm{Si}_{15} \mathrm{~B}_{10}$, which present low magnetostriction, exhibit the giant magnetoimpedance (GMI) phenomenon.

The GMI samples can be electrically modelled by a simple RL model of a resistor $R_{\text {sens }}(H)$ in series with an inductor $L_{\text {sens }}(H)$ as defined by equation (1).

$Z_{\text {sens }}(H)=R_{\text {sens }}(H)+j \omega L_{\text {sens }}(H)$,

where

$$
\begin{gathered}
R_{\text {sens }}(H)=\left|Z_{\text {sens }}(H)\right| \cos \theta_{\text {sens }}(H), \\
L_{\text {sens }}(H)=\frac{\left|Z_{\text {sens }}(H)\right| \sin \theta_{\text {sens }}(H)}{\omega}
\end{gathered}
$$

and $\theta_{\text {sens }}$ is the impedance phase of the GMI samples.

Experimentally the GMI phenomenon is induced by the application of an alternating current $(I)$ along the length of ferromagnetic samples, which are then submitted to an external magnetic field $(H)$, as shown in Figure 1. The impedance of

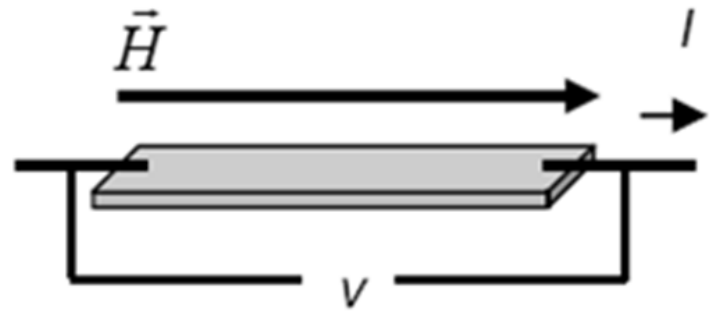

Figure 1. Typical measurement of the GMI effect: The difference of potential $(V)$ between the extremities of a GMI sample, submitted to an excitation current $(I)$, as a function of the magnetic field $(H)$.

GMI samples changes as a function of $H$, and $H$ can be inferred by measuring the difference of potential $(V)$ between the extremities of the GMI sample. Thus, the magnitude and phase values can be acquired via conventional impedance measurements using an adequate device [19, 21].

As mentioned in the introduction, there is a wide variety of external factors that can impact the GMI effect in a sample. For example, the frequency of the current that flows through the sample, $I_{a c}$, directly modifies the skin effect and therefore the GMI behavior. Also, the DC current, $I_{d o}$ is responsible for AGMI (Asymmetric Giant Magnetoimpedance) effects [27], while small changes in the chemical composition of the sample material can often have dramatic effects on GMI percentages [26]. As in most materials that are influenced by magnetic phenomena, GMI samples suffer from magnetic hysteresis [2], which certainly accounts as an obstacle for the construction of a magnetometer, and consequently should be carefully analyzed.

\section{DESCRIPTION OF THE SYSTEM}

Aiming to make the characterization process of the GMI samples more agile, it was developed an automatic characterization system [21]. As shown in Figure 2, the system is composed by six parts essential to its functionality: a Helmholtz coil for the generation of the external magnetic field; an LCR meter that electrically excites the sample and, simultaneously, measures its impedance; a current source $\left(I_{H}\right)$ to excite the Helmholtz coil; a polarity inverter to change the direction of the current source; digital outputs of a DAQ (Data Acquisition Device) module to control the polarity inverter; and, finally, the software developed in LabVIEW to serve as a virtual interface between the operator and the other modules. The selection of the experimental configurations in the LabVIEW software is made by the user. Specifically, the user defines, via a simple graphical interface, a list of experiments to carry out.

The uniaxial Helmholtz coil has 48 turns and a radius of 15 $\mathrm{cm}$ and it is responsible by generating the DC magnetic field used to excite the GMI samples, according to equation (4). This coil is powered by a DC current source (E3648, Agilent), controlled by a GPIB interface, which has a maximum power capacity of $100 \mathrm{~W}$ and a dual output, each one capable of providing $8 \mathrm{~V} / 5 \mathrm{~A}$ or $20 \mathrm{~V} / 2.5 \mathrm{~A}$. As it allows a broader range of magnetic fields during the characterization process of the GMI samples, the current source was used in the mode where $I_{\max }=5 \mathrm{~A}$ (corresponding to $H_{\max } \approx 14.4$ Oe inside the coil). To analyze the behaviour of GMI samples excited by higher magnetic fields, the system must be redesigned by 


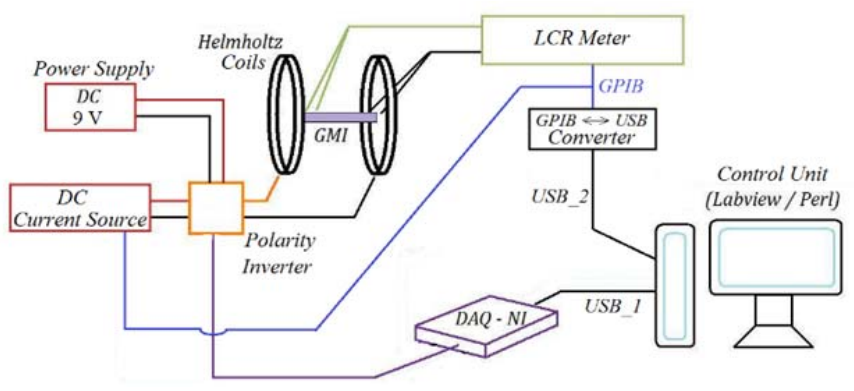

Figure 2. Basic setup of the automatic system for GMI sample characterization.

increasing the source power and/or the number of turns of the Helmholtz coil.

$$
\frac{H}{\text { Oe }}=2.88 \times \frac{I_{H}}{\mathrm{~A}} \text {. }
$$

The developed system also contains a polarity inverter to reverse the direction of the current that flows through the Helmholtz coils and, consequently, enabling to generate magnetic fields in both directions. As shown in Figure 2, this polarity inverter is powered by a $9 \mathrm{~V}$ voltage source and the polarity reversal is controlled by a TTL signal $\left(V_{\text {in }}\right)$ generated by an acquisition board (NI USB-6221, National Instruments). The electronic circuit designed for this polarity inverter, shown in Figure 3, consists of a double relay (ET2-B3M1S, NEC), able to support currents up to $25 \mathrm{~A}$ and with switching times of less than $5 \mathrm{~ms}$, junction diodes $D_{1}$ and $D_{2}(1 \mathrm{~N} 4001)$ and $n p n$ junction bipolar transistors $Q_{1}$ and $Q_{2}$ (BC547). The control voltage $V_{\text {in }}$ is not applied directly to the terminals of the relay coils because the acquisition board is not capable of providing more than $24 \mathrm{~mA}$ per digital output and the switching coils of the relays require currents of $50-80 \mathrm{~mA}$ to be magnetized. Therefore, the transistors $Q_{1}$ and $Q_{2}$ were employed to allow the proper excitation of the switching coils. In Figure 3, the terminals indicated by $F_{+}$and $F$ are, respectively, the connection points of the positive and negative terminals of the controlled current source. In turn, the outputs $H_{+}$and $H_{\text {. are, }}$ respectively, the connection points of the positive and negative

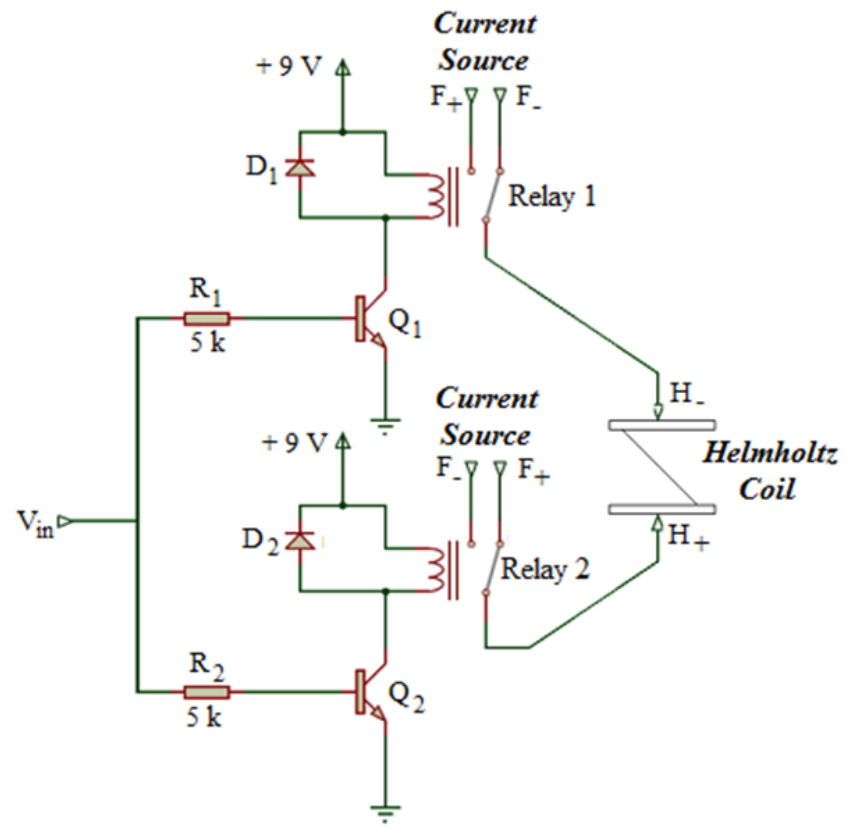

Figure 3. Schematic diagram of the electronic circuit developed for the polarity inverter. terminals of the Helmholtz coil.

The core of the system is a precision LCR meter (4285A, Agilent), responsible for measuring the impedance magnitude and phase of GMI sensor elements, for each magnetic field generated by the Helmholtz pair. The LCR meter is also responsible by the excitation of the GMI samples, being capable of generating currents with adjustable amplitudes (100 $\mu \mathrm{A}_{\text {rms }}$ to $\left.20 \mathrm{~mA}_{\mathrm{rms}}\right)$, frequencies $(75 \mathrm{kHz}$ to $30 \mathrm{MHz})$ and $\mathrm{DC}$ levels $(0$ to $100 \mathrm{~mA})$.

Finally, in Figure 2 it is also depicted a GPIB-USB converter (82357B, Agilent) used to allow the communication between the GPIB interfaces of the controlled current source and of the LCR meter and the USB bus of the computer which is running the developed LabVIEW software, responsible by controlling the entire measuring process and properly processing the acquired information.

At the end of each characterization test an Excel output file is automatically generated, containing a header with the parameters defined for the test and the measurement results of the impedance magnitude $\left|Z_{\text {sens }}(H)\right|$ and phase $\theta_{\text {sens }}(H)$ of the analyzed GMI sensors, as a function of the magnetic field $H$. In addition to the experimental measurements of magnitude and phase, these files also contain the mathematically calculated resistance $R_{\text {sens }}(H)$, equation (2), and inductance $L_{\text {sens }}(H)$, equation (3), of the GMI sensors, assuming that they can be electrically modelled as a resistance $R_{\text {sens }}(H)$ in series with an inductance $L_{\text {sens }}(H)$ as according to the electrical model defined by equation (1), which is useful to simulate the behaviour of the sensor elements in a SPICE program. It is noteworthy that, as magnetic sensors usually have hysteresis, the characterizations were performed in such a way to obtain the hysteresis curves of the samples. Then, the output files include graphs with hysteresis characteristics and respective averaged values.

The system was tested with ribbon-shaped samples having a chemical composition of $\mathrm{CO}_{70} \mathrm{Fe}_{5} \mathrm{Si}_{15} \mathrm{~B}_{10}$. The studied samples are of the LMI (Longitudinal Magnetoimpedance) type, which are much more sensitive (about 100 times) to the component of the magnetic field parallel to their lengths. Thus, it is recommended that, during the measurement process, the GMI ribbon be positioned in such a way that the Earth's magnetic field is perpendicular to its length. In this way, noise caused by the Earth's magnetic field is minimized.

As an example, consider the following experiment: a GMI sample of $\mathrm{CO}_{70} \mathrm{Fe}_{5} \mathrm{Si}_{15} \mathrm{~B}_{10}$ alloy with $5 \mathrm{~cm}$ length, $2 \mathrm{~mm}$ width and $60 \mu \mathrm{m}$ thickness is subject to a measurement temperature of $298 \mathrm{~K}$ and excited by a current with a DC level of $80 \mathrm{~mA}, 15$ $\mathrm{mA}$ amplitude and $1.5 \mathrm{MHz}$ frequency. Figure $4 \mathrm{a}$ show the details of a typical output Excel file, while Figures $4 \mathrm{~b}$ and $4 \mathrm{c}$ highlight, respectively, the hysteresis curves and the mean curves.

To recognize how each parameter affects the GMI behaviour it is required the analysis of a significant amount of experimental data, which demands long periods of time. Thus, aiming at improving the analysis of the output data, it was also developed a program named "Organize", written in Perl, which can be directly called by the LabVIEW interface of the main program. It processes the experimental data from multiple measurements and presents the results in a friendlier way, helping their interpretation by the user. The developed program allows the individual assessment of the influence of each of the parameters that affect the behaviour of the GMI samples. The user informs which parameter he wants to analyze and, based on this information, the program accesses the full database of 
(a)

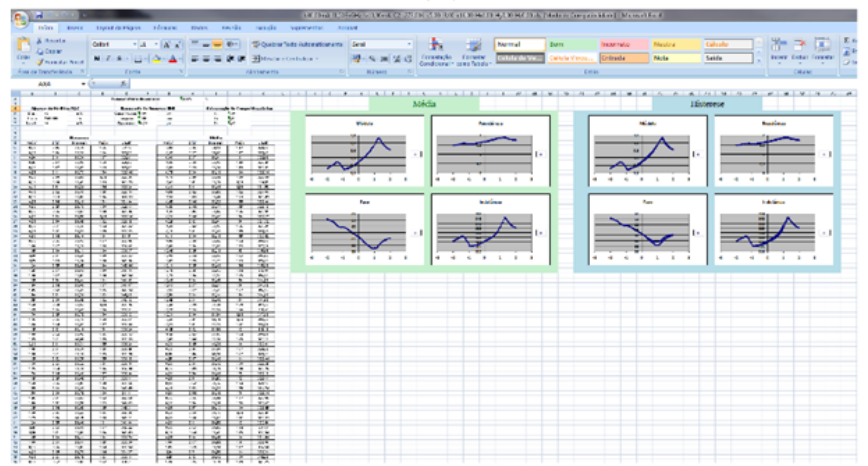

(b)

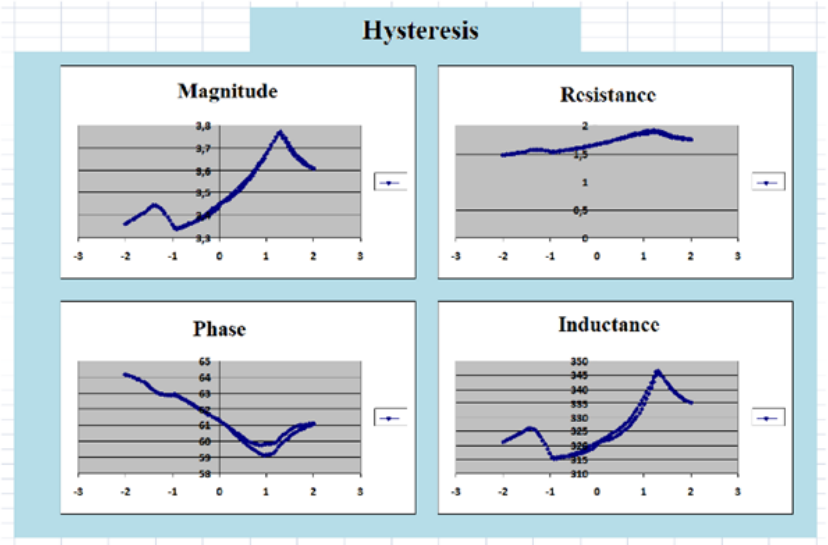

(c)

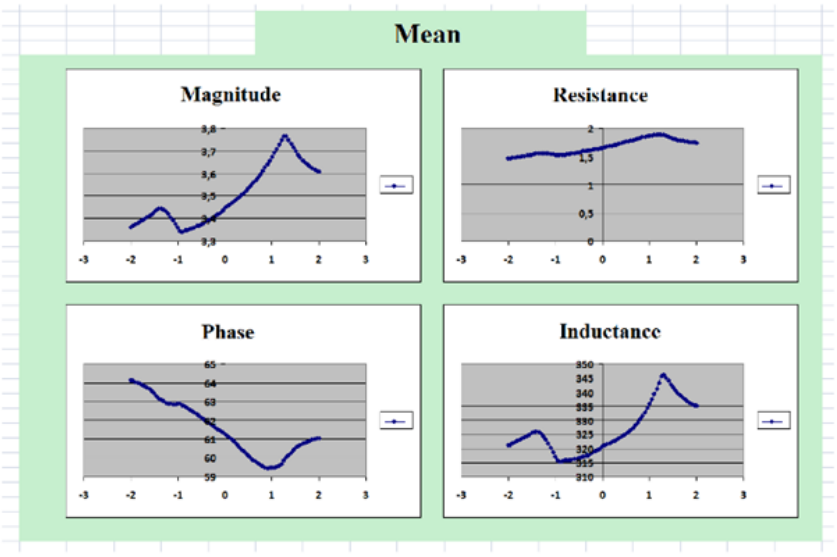

Figure 4. (a) Typical output file (b) Hysteresis curves of the impedance components of the sensor (c) Averaged curves of the impedance components of the sensor.

previously acquired measurements searching for identical configurations, where the only parameter differing among them is the one informed by the user. As a result it generates concise and simplified graphs, which highlights how the parameter of interest affects the behaviour of the GMI samples.

It should be noted that, after several measurements, it has been shown that, if certain techniques and circuitry are used, then the phase characteristic of GMI can be much more sensitive to magnetic fields than its counterpart magnitude characteristic [15-16, 19].

\subsection{Uncertainty Analysis}

Considering that, in the characterization studies, the impedance measurements were performed by the LCR meter 4285A (Agilent), the uncertainties of the impedance magnitude $\left(U_{\vartheta}\right)$ and phase $\left(U_{\theta}\right)$ measurements of the GMI samples are directly attributed to the uncertainties of the LCR meter, which are, respectively, defined in its operational manual as

$U_{z}(\%)= \pm\left(A_{n}+A_{c}\right) \times K_{t}$ and

$U_{\theta}($ degrees $)= \pm \frac{180 \times\left(A_{n}+A_{c}\right) \times K_{t}}{\pi \times 100}$,

where $A_{n}$ is the component of the uncertainty due to the equipment intrinsic characteristics, $A_{c}$ is the cable length factor and $K_{t}$ is the temperature factor.

The temperature factor $K_{t}$ is equal to one in the range of $18^{\circ} \mathrm{C}$ to $28^{\circ} \mathrm{C}$. The measurements were always performed within this temperature range, then it can be admitted that $K_{t}=1$.

For impedance magnitudes below $5 \mathrm{k} \Omega, A_{c}$ is given by

$A_{c}(\%)=\frac{f_{m}}{15}$,

where $f_{m}$ is the frequency, in $\mathrm{MHz}$, used to excite the sample.

On the other hand, knowing that all of the experimental measurements of the impedance of the GMI sensors returned magnitude values between $10 \mathrm{~m} \Omega$ and $100 \Omega$, the parameter $A_{n}$ is defined by the instrument maker as

$$
\begin{aligned}
A_{n}(\%)=N_{1} \% & +\left(\frac{f_{m}}{30}\right)^{2} \times 3 \%+ \\
& +\frac{50}{\left|Z_{m}\right|}\left[0.02 \%+\left(\frac{f_{m}}{30}\right) \times 0.1 \%\right] \times k_{i} \times k_{\text {osc }},
\end{aligned}
$$

where $\left|Z_{m}\right|$ is the absolute value of the measured impedance in ohms and $N_{1}$ is a frequency-dependent factor which can be equal to 0.15 - for $75 \mathrm{kHz}<f_{m}<200 \mathrm{kHz}$ or $3 \mathrm{MHz}<f_{m}<5$ $\mathrm{MHz}$; to 0.08 - for $200 \mathrm{kHz}<f_{m}<3 \mathrm{MHz}$; or to $0.30-f_{m}>5$ $\mathrm{MHz}$.

The constant $k_{i}$ is related to the integration time used by the LCR Meter, which can be set to short (30 ms), medium $(65 \mathrm{~ms})$ or long $(200 \mathrm{~ms})$. If the integration time is defined as long then $k_{i}=1$, else $k_{i}=2$. On the other hand, for impedance magnitudes between $10 \mathrm{~m} \Omega$ e $100 \Omega, k_{o s c}$ is given by

$k_{\text {osc }}=\left\{\begin{array}{l}1, \text { if }\left(20 / V_{o s c}\right) \leq 1 \\ \frac{20}{V_{o s c}}, \text { if }\left(20 / V_{o s c}\right)>1\end{array}\right.$,

where $V_{o s c}$ is the RMS value of the AC component of the voltage used to excite the samples, expressed in $\mathrm{mV}$.

Assuming a Gaussian distribution, the standard uncertainty of the impedance magnitude measurements $u_{z}$ is given by equation (10) and the one of the impedance phase $u_{\theta}$ is given by equation (11).

$$
\begin{aligned}
& u_{Z}(\Omega)=\frac{\left|Z_{m}\right| \times U_{z}(\%)}{2} \\
& u_{\theta} \text { (degrees) }=\frac{U_{\theta}}{2} .
\end{aligned}
$$

Figure $5 \mathrm{a}$ shows the expanded uncertainty of the impedance magnitude measurements $U_{z}(\%)$ and Figure 5b shows the expanded uncertainty of impedance phase measurements $U_{\theta}$ (degrees). Both of them are functions of the impedance magnitude of the samples and of the frequency of the current used to excite the samples. Those graphs were generated assuming that the integration time was defined as long, $k_{i}=1$, 
(a)
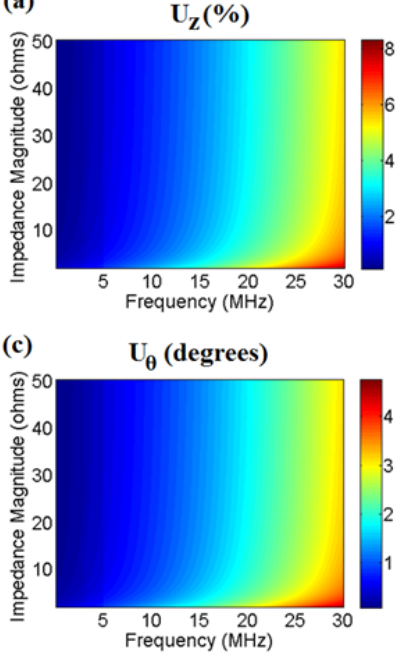

(b)
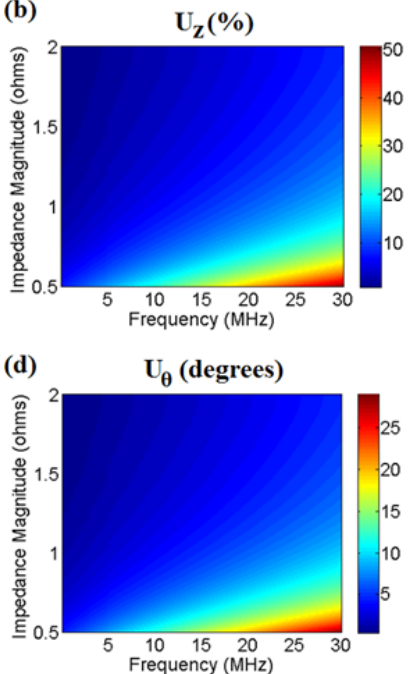

Figure 5. Impedance magnitude uncertainty $U_{z}(\%)$ and impedance phase uncertainty $U_{\theta}$ (degrees) as a function of the frequency $f_{m}$ and of the impedance magnitude $\left|Z_{m}\right|$. (a) $U_{z}(\%)$ for $75 \mathrm{kHz}<f_{m}<30 \mathrm{MHz}$ and $2 \Omega<$ $\left|Z_{m}\right|<50 \Omega$; (b) $U_{z}(\%)$ for $75 \mathrm{kHz}<f_{m}<30 \mathrm{MHz}$ and $0.5 \Omega<\left|Z_{m}\right|<2 \Omega$; (c) $U_{\theta}$ (degrees) for $75 \mathrm{kHz}<f_{m}<30 \mathrm{MHz}$ and $2 \Omega<\left|Z_{m}\right|<50 \Omega$; (d) $U_{\theta}$ (degrees) for $75 \mathrm{kHz}<f_{m}<30 \mathrm{MHz}$ and $0.5 \Omega<\left|Z_{m}\right|<2 \Omega$.

and that the amplitude of the excitation current was kept in $10.6 \mathrm{~mA}_{\mathrm{rms}}$, thus allowing to define $V_{o s c}=\left|Z_{m}\right| \times 10.6$.

By observing Figure 5, it can be noticed that the measurement uncertainties tend to decrease for low frequencies and high impedance values. On the contrary, high frequencies and small impedances increase the measurement uncertainties.

For the experimental measurements performed, the impedance measurement uncertainty $U_{z}$ of the results, obtained by applying equation (5) to the experimental data set, is always, at least, ten times smaller than its respective impedance value. Also, all of the measured impedance phase values presented uncertainties $U_{\theta \theta}$, obtained by applying equation (6), equal or smaller than $\pm 1^{\circ}$.

The standard uncertainty of the magnetic field $\left(u_{H}\right)$ generated by the Helmholtz pair is dependent of the standard uncertainty of the DC current source (Agilent, E3648A), which is equal to $\pm 2.0 \mathrm{~mA}$. Then, supposing, by simplicity, that the geometric configuration of the Helmholtz coils is satisfactorily close to the one considered on the theoretical model and knowing that the relation between the current and the magnetic field generated by the Helmholtz pair is given by equation (4), $u_{H}$ is expressed as

$u_{H}= \pm 2.88 \times u_{I}(A)= \pm 5.76 \mathrm{mOe}$.

Thus, the expanded uncertainty $U_{H}$, for a confidence level of $95.45 \%$, is

$U_{H}=2 \times u_{H}= \pm 11.52 \mathrm{mOe}$.

The smallest magnetic field step used for the GMI samples characterization was $0.1 \mathrm{Oe}$, which is about 10 times larger than $U_{H}$. It can be noticed that, in order to reduce the magnetic field step, it will be essential to improve the uncertainty of the current source.

\section{RESULTS}

The automated system has shown to be capable of acquiring a much larger amount of data than the manual process conventionally used, in the same timeframe. For instance, to generate the data shown in Figure 6 by means of a manual process, it would take approximately 19 hours of sequential

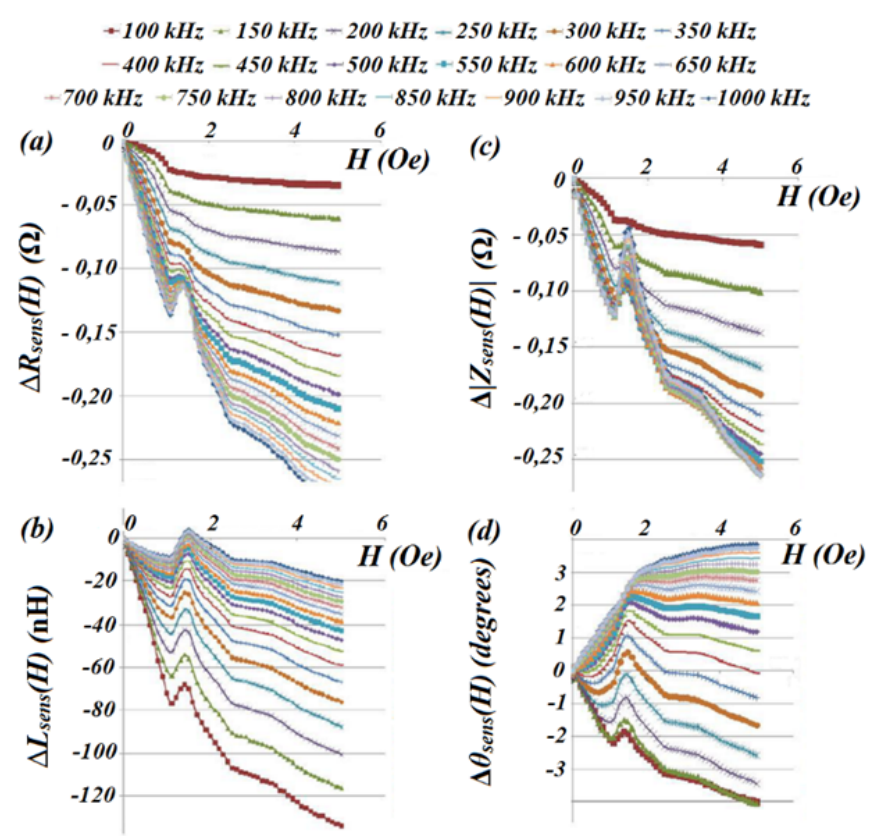

Figure 6. Example of an output file of the program "Organize", where the frequency is the parameter that the user wants to analyse. Variation of the (a) resistance, (b) inductance, (c) magnitude and (d) phase as a function of the magnetic field, for several frequencies.

experiments, while the automated system managed to acquire the same data in approximately 95 minutes. In other words, an experiment that would consume 1 hour using manual procedures takes 5 minutes using the current version of the automated characterization system.

In turn, Figure 6 illustrates an example of output file of the "Organize" program, where the parameter of interest, chosen by the user, is the frequency. The presented results are for a GMI sample of $\mathrm{CO}_{70} \mathrm{Fe}_{5} \mathrm{Si}_{15} \mathrm{~B}_{10}$ alloy with $5 \mathrm{~cm}$ length, $2 \mathrm{~mm}$ width and $60 \mu \mathrm{m}$ thickness. The measurements were performed at a room temperature of $298 \mathrm{~K}$ and, in all of them; the DC level was kept at $80 \mathrm{~mA}$ and the amplitude of the AC current at 15 $\mathrm{mA}$. This Figure shows four comparative graphs generated by the program, referring to variations, as a function of applied magnetic field, of the resistance $R_{\text {sens }}(H)$, inductance $L_{\text {sens }}(H)$, magnitude $\left|Z_{\text {sens }}(H)\right|$ and phase $\theta_{\text {sens }}(H)$ of the impedance GMI samples in relation to their respective values at $H=0$. The clear tendencies of the impedance components as a function of the frequency, shown in Figure 6, highlight the impact of this parameter on the GMI effect.

In this stage of research, the vast amount of data and variables that should be analyzed for optimizing the sensitivity of GMI sensor elements indicates the relevance of an automated system for the characterization of GMI effect.

\section{CONCLUSIONS}

The developed system for automated characterization of GMI samples, designed in the LabVIEW environment, allows a reliable and high speed identification of the impedance behaviour as a function of specific measurement parameters. The attained performance is essential for the use of this tool to identify the optimal operational point of GMI magnetometers.

The number of external parameters controlled by the current system can be expanded, incorporating other aspects discussed in the literature [2], and this expansion is an objective of the LaBioMet team. Also, while the current version has shown a 
great improvement in speed for the characterization measurements, optimization in the LabVIEW code itself is being discussed in order to increase its performance even more.

Finally, the large amount of raw information requires some type of data filtering and analysis, since the tendencies identified by human eye recognition need to be captured by optimization algorithms.

In this way, a future version of the automated characterization system would incorporate a feedback analysis to generate a new - and hopefully even more insightful - list of experiment configurations, aiming at reaching an optimal point, which is necessary for the development of a high sensitivity GMI magnetometer.

\section{ACKNOWLEDGEMENT}

We thank the Brazilian funding agencies CNPq, FAPERJ and FINEP, for their financial support, and Prof. Fernando Machado (Dept. of Physics/UFPE) for the GMI samples provided.

\section{REFERENCES}

[1] A. E. Mahdi, L. Panina, D. Mapps, "Some new horizons in magnetic sensing: high-Tc SQUIDs, GMR and GMI materials", Sensors and Actuators A: Physical 105 (2003) pp. 271-85.

[2] M. Phan, H. Peng, "Giant magnetoimpedance materials: Fundamentals and applications", Progress in Materials Science 53 (2008) pp. 323-420.

[3] R. Valensuela, M. Vazquez, A. Hernando, "A position sensor based on magnetoimpedance", J. Appl. Phys. 79 (1996) pp. 6549-91.

[4] H. Hauser, R. Steindl, C. Hausleitner, A. Pohl, J. Nicolics, "Wirelessly interrogable magnetic field sensor utilizing giant magnetoimpedance effect and surface acoustic wave devices", IEEE Instrum. Meas. 49 (2000) pp. 648-52.

[5] J. Lenz, A.S. Edelstein, "Magnetic sensors and their applications", IEEE Sensors Journal 6(3) (2006) pp. 631-49.

[6] Y. Honkura, "Development of amorphous wire type MI sensors for automobile use", J. Magn. Magn. Mater. 249 (2002) pp. 375-77.

[7] P. Delooze, L.V. Panina, D.J. Mapps, K. Ueno, H. Sano, "Effect of transverse magnetic field on thin film magnetoimpedance and application to magnetic recording", J. Magn. Magn. Mater. 272-276 (2004) pp. 2266-68.

[8] T. Uchiyawa, K. Mohri, H. Itho, K. Nakashima, J. Ohuchi, Y. Sudo, "Car traffic monitoring system using MI sensor built-in disk set on the road", IEEE Trans. Magn. 36 (2000) pp. 3670-72.

[9] D.J. Kim, D.G. Park, J.H. Hong, "Nondestructive evaluation of reactor pressure vessel steels using the giant magnetoimpedance sensor", J. Appl. Phys. 91(10) (2002) pp. 7421-23.

[10] H. Chiriac, M. Tibu, A.E. Moga, D.D. Herea, "Magnetic GMI sensor for detection of biomolecules", J. Magn. Magn. Mater. 293 (2005) pp. 671-73.

[11] G.V. Kurlyandskaya, M.L. Sanchez, B. Hernando, V.M. Prida, P. Gorria, M. Tejedor, "Giant magnetoimpedance based sensitive element as a model for biosensors", Appl. Phys. Lett. 82 (2003) pp. 3053-55.

[12] D.R. Louzada, E. Costa Monteiro, L.A.P. Gusmão, C.R. Hall Barbosa, "Medição não-invasiva de ondas de pulso arterial utilizando transdutor de pressão MIG", Proc. of IV Latin American Congress on Biomedical Engineering, 2007, Isla de Margarita, Venezuela, pp. 436-439.

[13] F. Pompéia, L.A.P. Gusmão, C.R. Hall Barbosa, E. Costa Monteiro, L.A.P. Gonçalves, F.L.A. Machado, "Ring shaped magnetic field transducer based on the GMI effect", Meas. Sci. Tech. 19 (2008) pp. 1-10.

[14] E.C. Silva, L.A.P. Gusmão, C.R.H. Barbosa, E.C. Monteiro, F.L.A. Machado, "Sensitivity improvement of GMI magnetic and pressure transducers for biomedical measurements", Braz. J. Biom. Eng. 27(2) (2011) pp. 79-89.

[15] E. Costa Silva, L.A.P. Gusmão, C.R. Hall Barbosa, E. Costa Monteiro, "Progress toward a hundredfold enhancement in the impedance phase sensitivity of gmi magnetic sensors aiming at biomagnetic measurements", Proc. of V Latin American Congress on Biomedical Engineering, 2011, Cuba, pp. 1-4.

[16] E.C. Silva, L.A.P. Gusmão, C.R.H. Barbosa, E.C. Monteiro, "Magnetic field transducers based on the phase characteristics of gmi sensors and aimed at biomedical applications", Proc. of 13th International Conference on Biomedical Engineering, 2009, Singapore, pp. 652-56.

[17] D. Robbes, C. Dolabdjian, S. Saez, Y. Monfort, G. Kaiser, P. Ciureanu, "Highly Sensitive Uncooled Magnetometers: State of the Art Superconducting Magnetic Hybrid Magnetometers, an alternative to SQUIDs?", IEEE Transactions on Applied Superconductivity 2(1) (2001) pp. 629-34.

[18] W. Andrä, H. Nowak, "Magnetism in medicine: a handbook", WILEY-VCH, Weinheim, 2007.

[19] E.C. Silva, L.A.P. Gusmão, C.R.H. Barbosa, E.C. Monteiro, F.L.A. Machado, "High sensitivity giant magneto-impedance (GMI) magnetic transducer: magnitude versus phase sensing", Measurement Science \& Technology 22(3) (2011) pp. 1-9.

[20] E. Costa Silva, M.M.B.R. Vellasco, C. Hall Barbosa, E. Costa Monteiro, L.A.P. Gusmão, "Modeling the sensitivity of GMI samples by neural networks", Controle \& Automação 23 (2012) pp. 636-48.

[21] J.H.C.C. Carneiro, E. Costa Silva, L.A.P. Gusmão, C.R.H. Barbosa, E. Costa Monteiro, "System for automatic characterization of giant magneto-impedance samples", Proc. of XX IMEKO World Congress, Sept. 9-14, 2012, Busan, Republic of Korea, pp. 1-5.

[22] L.A.P Gonçalves, J.M. Soares, F.L.A Machado, W.M. de Azevedo, "GMI effect in the low magnetostrictive $\mathrm{CoFeSiB}$ alloys", Physica B 384 (2006) pp. 152-54.

[23] H. Hauser, L. Kraus, P. Ripka, "Giant magnetoimpedance sensors", IEEE Instrumentation \& Measurement Magazine, 4(2) (2001) pp. 28-32.

[24] V. Knobel, K.R. Pirota, "Giant magnetoimpedance concepts and recent progress", Journal of Magnetism and Magnetic Materials 242 (2002) pp. 33-40.

[25] F.L.A. Machado, S.M. Rezende, "A theoretical model for the giant magnetoimpedance in ribbons of amorphous softferromagnetic alloys", Journal of Applied Physics 79 (1996) pp. 6958-60.

[26] K.C. Mendes, F.L.A. Machado, "Enhanced GMI in ribbons of Co70.4Fe4.6Si15B10 alloy", Journal of Magnetism and Magnetic Materials 177 (1998) pp. 111-12.

[27] F.L.A. Machado, A.R. Rodrigues, A.A. Puça, A.E.P. de Araújo, "Highly asymmetric Giant Magnetoimpedance", Materials Science Forum 302-303 (1999) pp. 202-8. 